\title{
Renormalization group in the infinite-dimensional turbulence: determination of the RG-functions without renormalization constants
}

\author{
L. Ts. Adzhemyan ${ }^{1}$, T. L. Kim¹ ${ }^{1}$ M. V. Kompaniets ${ }^{1}$, V. K. Sazonov ${ }^{1,2}$ \\ ${ }^{1}$ Department of Theoretical Physics, St. Petersburg State University, Uljanovskaja 1, \\ St. Petersburg, Petrodvorez, 198504 Russia \\ ${ }^{2}$ Institute of Physics, Department of Theoretical Physics, University of Graz, \\ Universitätsplatz 5, A-8010 Graz, Austria \\ l.adzhemyan@spbu.ru,mkompan@gmail.com, vasily.sazonov@uni-graz.at
}

PACS 05.10.Cc, 05.10.Gg, 05.45.Jn

DOI 10.17586/2220-8054-2015-6-4-461-469

\begin{abstract}
We calculate renormalization-group functions in the developed turbulence model for infinite dimensional space $d \rightarrow \infty$ using an operating method without renormalization constants. The renormalization fixed point and index $\omega$, obtained within the considered three loop approximation, are in agreement with previous calculations. The results demonstrate the efficiency of the method and the possibility of its complete automation, which is crucially important in higher order perturbation theory computations.
\end{abstract}

Keywords: turbulence, renormalization group (RG).

Received: 20 June 2015

\section{Introduction}

The explanation for the anomalous scaling in fully developed turbulence, which describes the deviations from the Kolmogorov phenomenological theory, is an actual problem of the modern statistical mechanics. Currently, the anomalous exponents have been calculated and anomalous scaling was obtained only in the simplified turbulence model - the rapid-change model of the passive scalar advection. It was shown in [1] that in spaces with large dimensions $d$, this model is reduced to the Kolmogorov theory and that the exponents of the anomalous scaling tends to zero, as $d \rightarrow \infty$. These exponents were calculated in [1] up to the first order in $1 / d$.

There are indications that in turbulence theory, based on the Navier-Stokes equations, the limiting dimension $d \rightarrow \infty$ plays a role of the critical dimension of the space, for which the Kolmogorov theory becomes valid [2]. For the analysis of this asymptotic theory the renormalization group method and $\varepsilon$-expansion were applied in [3]. The studies in [3] revealed significant simplifications in the limit $d \rightarrow \infty$. This allowed the authors to perform the three loop analytical calculations of the RG-fixed point and the index $\omega$ and then to guess the formula for these quantities analogous to the corresponding one in the Heisenberg model for the developed turbulence [4]. It would be highly interesting to verify the correctness of this formula, comparing its predictions with those of fourth order perturbation theory. However, such verification is not a trivial task, because of the number of diagrams dramatically increases with the increasing order of the perturbation theory (there are 1692 four-loop Feynman diagrams). Thus, the automation of the computational process is required. 
In the current work, we apply the method of automatic computations for the renormalization group functions developed in [5], [6] in application to three-loop calculations. The method described in [5], [6] allows one to represent the renormalization group functions without using the renormalization constants in terms of the non-singular in $\varepsilon$ integrals. All necessary terms of the $\varepsilon$-expansion can be found by expanding the integrands of these integrals into a Taylor series.

The automatic three-loop calculations confirm the $\omega$ index value obtained in [3]. The value for the charge in the fixed point differs from the corresponding one in [3], because of the utilization of different renormalization schemes.

\section{The model}

The microscopic model of the fully developed, homogeneous, isotropic turbulence of the incompressible viscous fluid or gas is usually described by the stochastic Navier-Stokes equation with a random driving force:

$$
\partial_{t} v_{i}=-\partial_{i} P-\left(v_{j} \partial_{j}\right) v_{i}+\nu_{0} \partial^{2} v_{i}+f_{i},
$$

where $v_{i}$ is the velocity field, $P$ and $\nu_{0}$ are the pressure and the kinematic viscosity, respectively, $f_{i}$ is the random force per unit mass. Equation (1) is complemented by the incompressibility condition $\partial_{i} v_{i}=0$, which leads to the transverseness of the velocity field and force. We assume for $f$ a Gaussian distribution with zero mean and correlator:

$$
\begin{gathered}
\left\langle f_{i}\left(t_{1}, \mathbf{x}_{1}\right) f_{j}\left(t_{2}, \mathbf{x}_{2}\right)\right\rangle \equiv D_{i j}^{f}\left(t_{1}-t_{2}, \mathbf{x}_{1}-\mathbf{x}_{2}\right), \\
D_{i j}^{f}(t, \mathbf{k})=\delta(t) P_{i j}(\mathbf{k}) d_{f}(k)
\end{gathered}
$$

where $P_{i j}(\mathbf{k}) \equiv \delta_{i j}-k_{i} k_{j} / k^{2}$ is the transverse projector. In the inertial interval of the wavenumbers $m \ll k \ll k_{\text {diss }}\left(m^{-1}=L\right.$ is the external turbulence scale, $k_{\text {diss }}$ is the dissipative scale) one can use the power-law model

$$
d_{f}(k)=D_{0} k^{4-d-2 \varepsilon} \theta(k-m),
$$

where $\varepsilon$ is analogous to the $4-d$ parameter in the Wilson theory of the phase transitions. The physical value of $\varepsilon$ is equal to 2 and corresponds to an ideal pumping of the energy into the system by infinitely-sized vortices.

According to the fundamental theorem [7], the stochastic equation (1) is equivalent to quantum field theory with a double set of transverse fields determined by the action:

$$
S_{0}=v^{\prime} D^{f} v^{\prime} / 2+v^{\prime}\left(-\partial_{t} v_{i}-(v \partial) v+\nu_{0} \partial^{2} v\right) .
$$

Here, all the necessary integrations and summations over indices are meant. The contribution of the pressure in (5) is omitted due to the transverseness of the auxiliary field $v^{\prime}$.

The diagrams of the perturbation theory determined by the action (5) contain ultraviolet (UV) divergences, when $\varepsilon \rightarrow+0$. The invariance of the action under the Galilean transformations results in the only singly divergent 1-irreducible correlation function, $\Gamma_{i j}^{(0)}=<v_{i} v_{j}^{\prime}>_{1-i r}$ (1-irreducible response function). To cancel divergences in this function, one needs the only one counterterm of the type $v^{\prime} \partial^{2} v$. The renormalized action is given by:

$$
S=v^{\prime} D^{f} v^{\prime} / 2+v^{\prime}\left(-\partial_{t} v_{i}-(v \partial) v+\nu Z_{\nu} \partial^{2} v\right)
$$

which is obtained from (5) by the multiplicative renormalization of the parameters:

$$
D_{0}=g_{0} \nu_{0}^{3}=g \mu^{2 \varepsilon} \nu^{3}, \quad \nu_{0}=\nu Z_{\nu}, \quad g_{0}=g \mu^{2 \varepsilon} Z_{g}, \quad Z_{g}=Z_{\nu}^{-3},
$$

where $\mu$ is the renormalization mass, $g$ is the dimensionless renormalized charge and the renormalization of the fields is not required. 
In the following, we use the renormalization scheme, analogous to the renormalization on the zero frequencies and moments, complemented by the extra condition $\mu=m$. Let $\Gamma_{i j}(\mathbf{k}, \omega)$ be the 1-irreducible response function calculated in accordance with action (6) with the renormalization constant $Z_{\nu}=1$. This function is proportional to the transverse projector:

$$
\Gamma_{i j}(\mathbf{k}, \omega)=P_{i j}(\mathbf{k}) \Gamma(k, \omega), \quad \Gamma(k, \omega)=\frac{\Gamma_{i i}(\mathbf{k}, \omega)}{d-1} .
$$

Let us now define a normalized function equal to unity in the zero (loop-less) approximation:

$$
\bar{\Gamma}(k, \omega)=\frac{\Gamma(k, \omega)}{-\nu k^{2}} .
$$

Then, for its renormalized version, we require the following normalization conditions:

$$
\left.\bar{\Gamma}^{R}\right|_{k=0, \omega=0, \mu=m}=1,
$$

namely, all diagrammatic contributions to the renormalized function $\bar{\Gamma}^{R}$ must be canceled out by the counterterms in the normalization point $k=0, \omega=0, \mu=m$. This condition defines the subtraction scheme and the form of the renormalization constant $Z_{\nu}$.

The Feynman diagrammatic technique, corresponding to the model (6), (3), (4), contains the following propagators, given in the $(\mathbf{k}, \mathbf{t})$-representation by:

$$
\begin{gathered}
<v_{i}\left(t_{1}\right) v_{j}\left(t_{2}\right)>=\frac{d_{f}(k)}{2 \nu k^{2}} \exp \left[-\nu k^{2}\left|t_{1}-t_{2}\right|\right] P_{i j}(\mathbf{k})=- \\
<v_{i}\left(t_{1}\right) v_{j}^{\prime}\left(t_{2}\right)>=\theta\left(t_{1}-t_{2}\right) \exp \left[-\nu k^{2}\left(t_{1}-t_{2}\right)\right] P_{i j}(\mathbf{k})=\square
\end{gathered}
$$

The interaction in (6) is represented by the triple vertex $-v^{\prime}(v \partial) v=v_{j}^{\prime} V_{j s} v_{s} v_{l}$ with the vertex factor:

$$
V_{j s l}=i k_{s} \delta_{j l}=-1
$$

where $k_{s}$ is the momentum argument of the field $v^{\prime}$. The crossed endpoint in (13) corresponds to the field $v^{\prime}$, the endpoint marked by the bold dot corresponds to the field $v_{s}$ contracted with $i k_{s}$ and the plain line stands for the field $v_{l}$.

We represent the perturbation series for the function $\bar{\Gamma}$ as:

$$
\bar{\Gamma}(k, \omega, m, \mu)=\sum_{n \geqslant 1} u^{n} \mu^{n \varepsilon} \sum_{i} \chi_{n}^{(i)}(k, \omega, m), u \equiv \frac{S_{d} g}{(2 \pi)^{d}},
$$

where the $i$-summation runs over all $n$-loop diagrams of the function $\bar{\Gamma}$. For convenience, we introduce a normalized charge $u$, in which $S_{d}$ is the surface area of the unit sphere in the $d$-dimensional space.

\section{Renormalization group equations, RG-functions expressed in terms of the renormalized Green functions}

In our renormalization scheme the renormalization constants $Z_{\nu}$ and $Z_{g}$ (similar to the MS scheme) depend only on the space dimension $d$ and parameter $\varepsilon$ and do not depend on the ratio $m / \mu$. The equations of the renormalization group are obtained from the independence 
of the non-renormalized Green functions on the parameter $\mu$ at fixed $\nu_{0}$ and $g_{0}$. The RGequations look exactly the same as in the MS scheme [8]. In particular, the equation for the 1-irreducible function $\Gamma^{R}$ is given by:

$$
\left(\mu \partial_{\mu}+\beta(g) \partial_{g}-\gamma_{\nu}(g) \nu \partial_{\nu}\right) \Gamma^{R}=0
$$

where

$$
\gamma_{i}(g)=\frac{-2 \varepsilon g \partial_{g} \ln Z_{i}}{1+g \partial_{g} \ln Z_{g}}, \quad \beta(g)=-g\left(2 \varepsilon+\gamma_{g}\right)=-g\left(2 \varepsilon-3 \gamma_{\nu}\right)
$$

The latter equation in (16) is a consequence of the relation between the renormalization constants $Z_{g}$ and $Z_{\nu}$ in (7). The equations (16) define the $\beta$ and $\gamma_{\nu}$ RG-functions in terms of the renormalization constants. These functions are finite and do not contain poles in $\varepsilon$, because of the renormalizability of the theory. However, the required preliminary calculation of the singular in $\varepsilon$ renormalization constants is complicated, especially for the numerical evaluation of $\beta$ and $\gamma_{\nu}$. Using equation (15), we express these RG-functions in term of the renormalized Green function $\Gamma^{R}$.

First of all, we derive the RG-equation for the normalized function $\bar{\Gamma}^{R}$. Employing (9) and (10) we find:

$$
\left(\mu \partial_{\mu}+\beta(g) \partial_{g}-\gamma_{\nu}(g) \nu \partial_{\nu}\right) \bar{\Gamma}^{R}=\gamma_{\nu} \bar{\Gamma}^{R}
$$

Considering this equation in the normalization point $k=0, \omega=0, \mu=m$ and taking into account that:

$$
\left.\bar{\Gamma}^{R}\right|_{k=0, \omega=0}(m, \mu, \nu)=\left.\bar{\Gamma}^{R}\right|_{k=0, \omega=0}(m / \mu),\left.\quad \partial_{g} \bar{\Gamma}^{R}\right|_{k=0, \omega=0, \mu=m}=0,
$$

we obtain:

$$
\gamma_{\nu}(g)=-\left.\left(m \partial_{m} \bar{\Gamma}^{R}\right)\right|_{k=0, \omega=0, \mu=m} .
$$

In (19), the RG-function $\gamma_{\nu}$ is written in terms of the renormalized function $\bar{\Gamma}^{R}$. Usually, computation of the renormalized functions involves calculation of the divergent in $\varepsilon$ renormalization constants. For calculations involving finite objects, we need to take into account the counterterms by the $R$-operation, acting on the diagrams of the basic action, where $Z_{\nu}=1$,

$$
\Gamma^{R}=R \Gamma=(1-K) R^{\prime} \Gamma .
$$

Here, the $R^{\prime}$-operation eliminates divergences in the subgraphs of diagrams and the operation $(1-K)$ removes the remaining superficial divergence. The $R^{\prime}$-operation can be expressed as $[9]$ :

$$
R^{\prime} \Gamma=\prod_{j}(1-K)_{j} \Gamma,
$$

where, for each diagram from $\Gamma$, the product runs over all its divergent subgraphs. The renormalization operation (20) eliminates the divergences in the function $\Gamma^{R}$ as a whole and separately in each diagram.

The formal UV-divergence index of the 1-irreducible function $\left\langle v v^{\prime}>_{1-i r}\right.$ equals 2 . This leads to the possible counterterms of the $k^{2}$ and $i \omega$ types. However, as is seen from (13), the external leg $v^{\prime}$ of this function is always multiplied by the external moment $k$, therefore, only the divergence of the $k^{2}$-type remains. In our renormalization scheme, this corresponds to the following subtraction operations for the whole function $\bar{\Gamma}$ and for the 1-irreducible subgraphs of diagrams $\chi_{n}^{(i)}$ from (14), respectively:

$$
\begin{array}{r}
(1-K) \bar{\Gamma}(k, \omega, m, \mu)=\bar{\Gamma}(k, \omega, m, \mu)-\left.\bar{\Gamma}\right|_{k=0, \omega=0, \mu=m}, \\
(1-K) \chi_{j}\left(k_{j}^{2}, \omega_{j}, m\right)=\chi_{j}\left(k_{j}^{2}, \omega_{j}, m\right)-\left.\chi_{j}\right|_{k_{j}=0, \omega=0},
\end{array}
$$


where $k_{j}$ and $\omega_{j}$ are the moment and frequency incoming to the subgraph $\chi_{j}$. The subtraction of the divergences in the subgraphs leads to the finite integrals corresponding to the renormalized Feynman diagrams.

Note that the dimensionless counterterm $\left.\bar{\Gamma}\right|_{k=0, \omega=0, \mu=m}$ does not depend on $m$, then, taking into account (19), we obtain:

$$
\gamma_{\nu}(g)=-\left.\left(m \partial_{m} R \bar{\Gamma}\right)\right|_{k=0, \omega=0, \mu=m}=-\left.\left(m \partial_{m} R^{\prime} \bar{\Gamma}\right)\right|_{k=0, \omega=0, \mu=m} .
$$

Substituting expansion (14) in (23), we find

$$
\gamma_{\nu}(g)=\sum_{n \geqslant 1} u^{n}\left(\gamma_{\nu}\right)_{n}, \quad\left(\gamma_{\nu}\right)_{n}=-\left.m^{-2 n \varepsilon} \sum_{i}\left(m \partial_{m} R^{\prime} \chi_{n}^{(i)}\right)\right|_{k=0, \omega=0, \mu=m} .
$$

This is the main relation for the calculation of the RG-function $\gamma_{\nu}$.

\section{Large $d$ limit}

Let us consider the diagrams in the momentum representation in the spherical coordinate system. Then, the dimension of the space $d$ enters into the integration measure $\int_{0}^{\infty} d k k^{d-1} \int_{0}^{\pi} d \theta(\sin \theta)^{d-2} \ldots$ and into the lines $v v$ of diagrams as $k^{2-d-2 \varepsilon}$. The number of $v v$-lines in diagrams of the function $\Gamma$ coincides with the number of loops, consequently the pure integration momenta can be always associated with $v v$-lines. Then, the factor $\theta(k-m) k^{2-d-2 \varepsilon}$ from (4) changes $\int_{0}^{\infty} d k k^{d-1}$ to $\int_{m}^{\infty} d k k^{1-2 \varepsilon}$ and the dependence on $d$ in the radial part disappears. When $d \rightarrow \infty$, the angular weight $(\sin \theta)^{d-2}$ has a sharp maximum at $\theta=\pi / 2$. Since $\cos (\pi / 2)=0$, the inner products of the different internal integration moments vanish. Then, in the leading approximation at $d \rightarrow \infty$ one may consider the internal integration moments to be orthogonal to each other and to the external moment $p$. In this approximation, the integrands do not depend on angles and the angular integrations give a factor $S_{d}$, included in the definition of the charge $u$ (14). The latter charge is finite in the renormalization group fixed point.

Therefore, the main contribution to the Green functions at $d \rightarrow \infty$ is given by the diagrams without the inner products, which drastically decreases the number of diagrams. In the one loop approximation from four diagrams in Fig. 1, only the first one gives the non-zero contribution:

$$
\left.\chi_{1}^{(1)}\right|_{k=0, \omega=0}=\int_{m}^{\infty} d k \frac{k^{1-2 \varepsilon}}{4 k^{2}} .
$$

Then, from equation (24), we find the one-loop expression for the anomalous dimension $\gamma_{\nu}$ :

$$
\left(\gamma_{\nu}\right)_{1}=-\left.m^{2 \varepsilon} m \partial_{m} \chi_{1}^{(1)}\right|_{k=0, \omega=0}=\frac{1}{4} .
$$
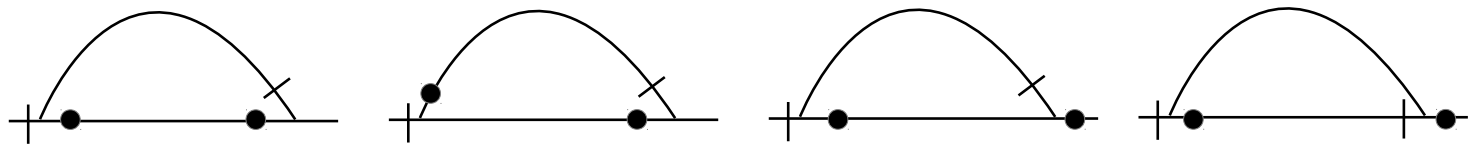

FIG. 1. One-loop diagrams.

In the two-loop approximation in the leading order for large $d$, only 6 of the 120 diagrams contribute. Let us consider the diagram in Fig. 2, as an example. Its integration over time gives:

$$
\left.\chi_{2}^{(1)}\right|_{k=0, \omega=0}=\frac{-1}{32} \int_{m}^{\infty} d k k^{1-2 \varepsilon} \int_{m}^{\infty} d q q^{1-2 \varepsilon} k^{2}\left(\frac{2}{k^{4}\left(k^{2}+q^{2}\right)}+\frac{1}{k^{2}\left(k^{2}+q^{2}\right)^{2}}\right) .
$$




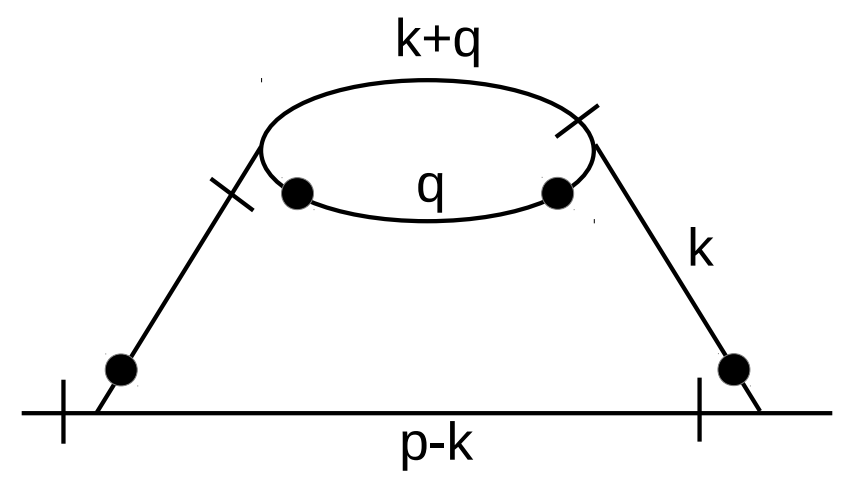

Fig. 2. Example of the two-loop diagram.

This diagram has a divergent subgraph with the circulating (internal) moment $q$ and external moment $k$. The corresponding divergence of the integral over $q$ at $\varepsilon=0$ is logarithmic and localized in the first summand in (27). Acting on this subgraph by the subtraction operation $(1-K)$ and using $(21)$, we obtain:

$$
\begin{aligned}
\left.R^{\prime} \chi_{2}^{(1)}\right|_{k=0, \omega=0} & =\frac{-1}{32} \int_{m}^{\infty} d k k^{1-2 \varepsilon}\left\{\int_{m}^{\infty} d q q^{1-2 \varepsilon} \frac{k^{2}}{k^{2}\left(k^{2}+q^{2}\right)^{2}}+\right. \\
& \left.+\left[\int_{m}^{\infty} d q q^{1-2 \varepsilon} \frac{2 k^{2}}{k^{4}\left(k^{2}+q^{2}\right)}-\int_{\mu}^{\infty} d q q^{1-2 \varepsilon} \frac{2 k^{2}}{k^{4} q^{2}}\right]\right\} .
\end{aligned}
$$

In accordance with (24), the contribution of this diagram to the $\left(\gamma_{\nu}\right)_{2}$ is given by:

$$
\begin{aligned}
-\left.\left(\left.\mu^{4 \varepsilon} m \partial_{m} R^{\prime} \chi_{2}^{(1)}\right|_{k=0, \omega=0}\right)\right|_{\mu=m}= & \frac{-1}{32} \int_{1}^{\infty} d q q^{1-2 \varepsilon}\left[\frac{2}{1+q^{2}}-\frac{2}{q^{2}}+\frac{1}{\left(1+q^{2}\right)^{2}}\right]+ \\
& -\frac{1}{32} \int_{1}^{\infty} d k k^{1-2 \varepsilon}\left[\frac{2}{k^{2}\left(1+k^{2}\right)}+\frac{1}{\left(1+k^{2}\right)^{2}}\right] .
\end{aligned}
$$

Changing the variables, as $q^{2}=x$ and $k^{2}=x$ and joining the integrals, we rewrite (29) as:

$$
-\left.\left(\left.\mu^{4 \varepsilon} m \partial_{m} R^{\prime} \chi_{2}^{(1)}\right|_{k=0, \omega=0}\right)\right|_{\mu=m}=-\frac{1}{32} \int_{1}^{\infty} d x \frac{x^{-\varepsilon}}{(x+1)^{2}} .
$$

The calculation of the remaining two loop diagrams leads to

$$
\left(\gamma_{\nu}\right)_{2}=\frac{1}{16} \int_{1}^{\infty} d x \frac{x^{-\varepsilon}}{(x+1)^{2}}
$$

In the MS-scheme the RG-functions do not depend on $\varepsilon$. In our scheme, the one loop result (26) is also independent of $\varepsilon$. The dependence on $\varepsilon$ of the two loop result can be found by expanding the integrand of (31) into the Taylor series. The number of the needed terms of this series is determined by the global desired precision. For the third order expansion in $\varepsilon$, taking into account that the charge $u$ in the fixed point $u_{*}=O(\varepsilon)$, it is necessary to know two following terms

$$
\left(\gamma_{\nu}\right)_{2}=\frac{1}{32}(1-2 \varepsilon \ln 2)+O\left(\varepsilon^{2}\right)
$$

Next, we consider an example of three-loop diagram calculation.

When the integration over time is performed, the diagram in Fig. 3 is given by the integral:

$$
\left.\chi_{3}^{(1)}\right|_{k=0, \omega=0}=\frac{1}{156} \int_{m}^{\infty} d k k^{1-2 \varepsilon} \int_{m}^{\infty} d q q^{1-2 \varepsilon} \int_{m}^{\infty} d s s^{1-2 \varepsilon} \frac{1}{k^{2}\left(k^{2}+q^{2}\right)\left(k^{2}+q^{2}+s^{2}\right)} .
$$


The diagram in Fig. 3 contains two divergent subgraphs: the one-loop subgraph with internal circulating momentum $s$ and external moments $k$ and $q$ ("subgraph 1") and the two-loop subgraph with internal circulating moments $q$ and $s$ and external momentum $k$ ("subgraph 2 "). The action of the $R^{\prime}$-operation is reduced to the consistent application of two subtraction operations $(1-K)_{2}(1-K)_{1}$ on the subgraphs in an arbitrarv order. Then, from (33), we

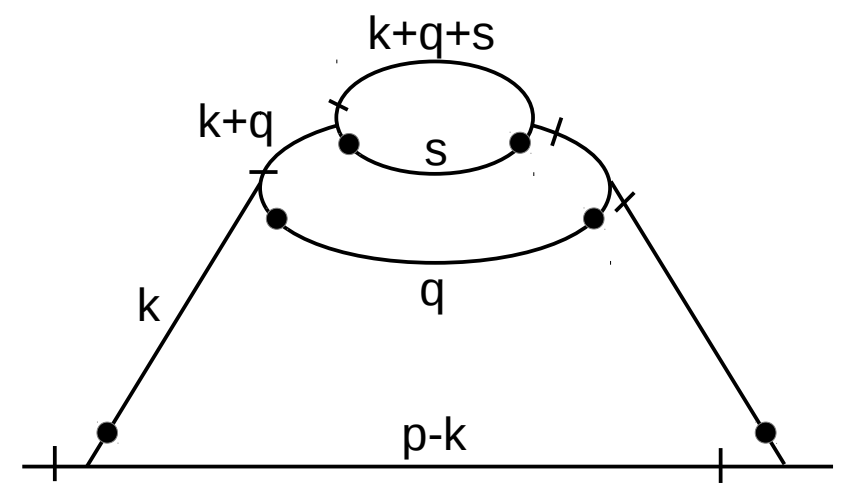

FIG. 3. Example of the three-loop diagram.

obtain:

$$
\begin{array}{r}
\left.R^{\prime} \chi_{3}^{(1)}\right|_{k=0, \omega=0}=\frac{1}{156} \int_{m}^{\infty} d k k^{-1-2 \varepsilon}\left\{\int _ { m } ^ { \infty } d q \frac { q ^ { 1 - 2 \varepsilon } } { ( k ^ { 2 } + q ^ { 2 } ) } \left[\int_{m}^{\infty} d s \frac{s^{1-2 \varepsilon}}{\left(k^{2}+q^{2}+s^{2}\right)}-\right.\right. \\
\left.\left.-\int_{\mu}^{\infty} d s \frac{s^{1-2 \varepsilon}}{s^{2}}\right]-\int_{\mu}^{\infty} d q \frac{q^{1-2 \varepsilon}}{q^{2}}\left[\int_{\mu}^{\infty} d s \frac{s^{1-2 \varepsilon}}{\left(q^{2}+s^{2}\right)}-\int_{\mu}^{\infty} d s \frac{s^{1-2 \varepsilon}}{s^{2}}\right]\right\} .
\end{array}
$$

Here, the first two summands are the results of the action of the operation $(1-K)_{1}$ on the one-loop subgraph; the third and fourth summands are the results of the action of $(1-K)_{2}$ on the whole diagram after the action of $(1-K)_{1}$. Differentiating (34) with respect to $m$, one can easily see that $-\left.\left(\left.\mu^{6 \varepsilon} m \partial_{m} R^{\prime} \chi_{3}^{(1)}\right|_{k=0, \omega=0}\right)\right|_{\mu=m}=0$, i.e. this diagram does not contribute to $\left(\gamma_{\nu}\right)_{3}$.

The total amount of the three-loop diagrams is 4080 and only 83 of those survive in the limit $d \rightarrow \infty$. Their contribution is given by:

$$
\left(\gamma_{\nu}\right)_{3}=\frac{1}{512}(7+6 \ln 2)+O(\varepsilon) .
$$

Therefore, from (24), (26), (32) and (35), it follows that the anomalous dimension $\gamma_{\nu}$ in the three-loop approximation is:

$$
\gamma_{\nu}=\frac{u}{4}+\frac{u^{2}}{32}(1-2 \varepsilon \ln 2)+\frac{u^{3}}{512}(7+6 \ln 2)+O\left(u^{4}\right) .
$$

Substituting (36) into the $\beta$-function (16) and solving the equation $\beta\left(u_{*}\right)=0$, we find the value of the charge in the fixed point in terms of the $\varepsilon$-expansion

$$
u_{*}=\frac{8 \varepsilon}{3}-\frac{8 \varepsilon^{2}}{9}-\frac{4 \varepsilon^{3}}{9}(1-2 \ln 2) .
$$

According to (16), (36) and (37), the index $\omega=\beta^{\prime}\left(u_{*}\right)$ is:

$$
\omega=2 \varepsilon+\frac{2}{3} \varepsilon^{2}+\frac{10}{9} \varepsilon^{3}
$$

The latter expression coincides with one obtained in [3]. 


\section{Conclusions}

We have performed calculations for the anomalous dimension $\gamma_{\nu}$ and exponent $\omega$ in the three-loop approximation in the fully developed turbulence model for large numbers of dimensions in space, by determining the of RG-functions without renormalization constants. Our calculations demonstrated the efficiency of the applied method. Its main advantage is that for the computation of the $n$-loop results, one needs to evaluate the set of the $(n-1)$ dimensional integrals, free from the singularities in $\varepsilon$. The computation procedure can be automated with relative ease. The main difficulty in higher order perturbation theory calculations is in the rapidly increasing number of diagrams with vector fields and the triple interaction vertex. In the considered limit, $d \rightarrow \infty$, the number of the diagrams is notably decreased. Thus, in four loops, only 1692 diagrams remain of the original 417872.

The significant decrease in the number of diagrams may indicate that the $\varepsilon$-expansion of the function $\gamma_{\nu}(\varepsilon)$ has a finite radius of convergence in the limit $d \rightarrow \infty$, in contrast to the general case, when one observes a factorial growth of the corresponding series coefficients. A similar thing occurs in phase transition theory when the anomalous dimensions $\gamma(\varepsilon, n)$ is calculated. Here $\varepsilon=4-d$, where $d$ is the dimension of the space and $n$ is the number of the field components. At fixed $n$ the coefficients of the $\varepsilon$-expansion grow factorially, but the coefficients of the $(1 / n)$-expansion have a finite radius of the convergence, as the series in $\varepsilon$ [8]. Currently, in turbulence theory only the third order in $\varepsilon$ at $d \rightarrow \infty$ of the double $(\varepsilon, 1 / d)$-expansion is known. However, the results obtained in the current work and in [3] have revealed some simplicity for this expansion. It is seen from the equations (37) and (38) that the irrational contribution to $u_{*}$ (the charge at the fixed point depends on the renormalization scheme) with $\ln 2$ disappearing in the physical quantity of the index $\omega$. The contributions of some particular diagrams proportional to $\ln ^{2} 2, \pi^{2}$, dilog(3/2), typical for the series of critical exponents in critical dynamics models, are all canceled out in the total sum of diagram. The coefficients of (38) are rational numbers. This gives the possibility to sum the series and find the function $\gamma_{\nu}(\varepsilon)$ at $d \rightarrow \infty$ without using the $\varepsilon$-expansion.

\section{Acknowledgements}

The work of LTsA, TLK and MVK was supported by Saint-Petersburg State University (project 11.38.185.2014). VKS acknowledges the support by the Austrian Science Fund FWF Grants Nr. I 1452-N27 and Nr. P21970-N16.

\section{References}

[1] M. Chertkov, G. Falkovich, I. Kolokolov, and V. Lebedev, Normal and anomalous scaling of the fourth-order correlation function of a randomly advected passive scalar. Phys. Rev. E, 1995, 52, 4924. M. Chertkov and G. Falkovich, Anomalous Scaling Exponents of a White-Advected Passive Scalar. Phys. Rev. Lett., 1996, 76, P. 2706.

[2] J.-D. Fournier, U. Frisch, H. A. Rose. Infinite-dimensional turbulence. J. Phys. A, 11(1), 1978, P. 187198.

[3] L. Ts. Adzhemyan, N. V. Antonov, P. B. Gol'din, T. L. Kim and M. V. Kompaniets. Renormalization group in the infinite-dimensional turbulence: third-order results, Journal of Physics A: Mathematical and Theoretical, 2008, 41(49), P. 495002.

[4] L.Ts. Adzhemyan, N.V. Antonov, Renormalization group in turbulence theory: Exactly solvable Heisenberg model. Theoretical and Mathematical Physics, 115, 1098, P. 562-574.

[5] L.Ts. Adzhemyan, M.V. Kompaniets, S.V. Novikov, V.K. Sazonov. Representation of the $\beta$-function and anomalous dimensions by nonsingular integrals: Proof of the main relation, Theoretical and Mathematical Physics, 2013, 175, P. 717-726. 
[6] L.Ts. Adzhemyan, M.V. Kompaniets, Five-loop numerical evaluation of critical exponents of the $p h i^{4}$ theory. Journal of Physics: Conference Series, 2014, 523, P. 012049.

[7] P.C. Martin, E.D. Siggia, H.A. Rose. Statistical Dynamics of Classical Systems. Phys.Rev., 1973, A8, P. 423.

[8] A. N. Vasil'ev The Field Theoretic Renormalization Group in Critical Behavior Theory and Stochastic Dynamics (Routledge Chapman \& Hall 2004); ISBN 978-0-415-31002-4

[9] O. I. Zavialov, Renormalized Quantum Field Theory, (Dordrecht :Kluwer, 1990). 